\title{
Jardín Infantil y su influencia en el rendimiento de coordinación visomotora, discriminación auditiva y lenguaje, medidos con una prueba de funciones básicas
}

OLCA BERDICEWSKI ${ }^{\circ}$ NEVA MILICIC **

InTRoDuccion, E] Jardín Infantil se concibe como una de las agencias de socializáción que provee nuestra cultura. Al no formar parte de la enseñanza abligatoria, es una oportunidad a la que no todos los niños tienen acceso.

Cabría esperar, dada la ejercitación de destrezas que posibilita el jardín, algunas diferencias entre niños que han tenido jardín respecto a aquéllos que no han pasado por dicha experiencia. Estudios de Wellman (1940) cit. cn Salvat (1972) comprueban una elcvación de 7 puntos en el coeficiente intelectual en niños que han asistido a la escuela maternal.

La existencia de sectores con problemas de privación psicosocial, o en condiciones desventajosas, determina problemas cn la adaptación de estos grupos a las exigencias que plantea la sociedad en su conjunto. Así, por ejemplo, en la educación, las exigencias o patrones que se hacen al nin̄o corresponden más bien a los niños de scctores medios y ello explica que se haya podido comprobar diferencias en el rendimiento escolar en los niños de diferentes estratos socioeconómicos (Milicic-Tshorne, 1974).

En psicología, desde hace tiempo, viene destacándose la importancia de las primeras cxperiencias en el desarrollo (Spitz, 1966; Bowly, 1965) y se ha planteado que las carencias de estimulación en los primeros estadios del desarrollo generan defjcits difíciles de superar.

Es así como se ha planteado la necesidad de una estimulación precoz, especialmente dirigida a los sectores de la población en condición desventajosa.

\footnotetext{
* Psicólogo - Master en Educación - Servicio de Neurologra y Psiquiatría, Hospital L. Calvo Mackcnna. Deptos. Pediatría y Psicología, Sedc Oriente U. de Chile - Escuela de Educación, Universidad Calólica de Chile.

* Psicalóso - Master en Eclucación - Depto. Esicología, Sedi Oriente U. de Chile - Escuela de Educación. Universidad Católica de Chile. Escuela Psicología U. Calólica, Stgo.
}

En nuestro medio no contamos en este momento con programas masivos de estimulación precoz, si bien algunos autores vienen planteándose el diseño de estos programas (Lira y Rodríguez, 1974). Quizás la única alternativa previa de la enseñanza sistemática la constituyen los jardines infantiles, que no alcanzan a cubrir sino un pequeño porcentaje de la población total.

Dentro de este enfoque, nos interesaba ver en qué medida la asistencia a Jardín Infantil, influiría en el puntaje de los niños en tres funciones básicas para cl rendimiento escolar, como son: lenguaje, coordinación visomotora y discriminación auditiva.

Elegimos estas tres funciones por contar con un instrumento de medición construido y analizado experimentalmente en nuestro país.

\section{MATERIAL Y METODO}

El instrumento utilizado en esta investigación es Ia Prueba de Funciones Básicas de Berdicewski-Milicic (1974 a). Es un test de papel-lápiz con ítens de tipo objetivo, que se aplica colectivamente. Mide tres funciones básices, a saber: Coordinación Visomotora, Discriminación Auditiva y Lenguaje. Dicha prueba, estudiada cxperimentalmente en una muestra de 370 sujetos tiene una confiabilidard global de 0.82 ; índice de consistencia interna estimado con el método Kuder-Richardson No 20 (1973).

La validez experimental del test se ha estudiado por el momento desde dos puntos de vista; uno referente a la validez predictiva; el otro como estudio de la validez concurrente. En relación a la validez predictiva, la Prueba de Funciones Básicas demostró que se relaciona con rendimiento en lectura y escritura en primer año. Como 
PROMEDIOS Y DESVIACIONES TIPICAS (STANDARD) EN LA PRUEBA DE FUNCIONES BASICAS, DE TRES GRUPOS DE NINOS DE NIVEL SOCIOECONOMICO ALTO, MEDIO Y BAJO, SEGUN HAYAN TENIDO O NO EXPERIENCIA EN JARDIN INFANTIL

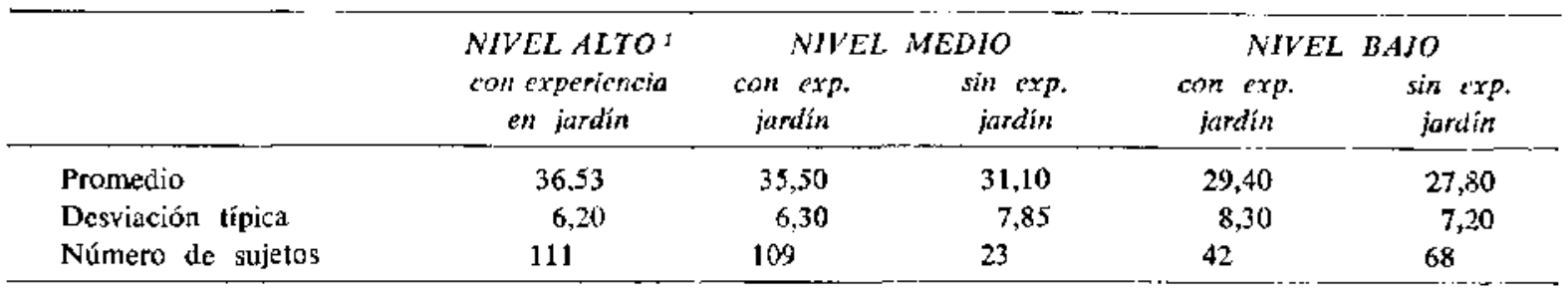

' El nivel alto no tiene subgrupo sin experiencia en jardín.

elemento de validación se utilizó la clasificación hecha por el profesor, seis meses después de haber sido aplicado el test. Así, los niños clasificados por el profesor con rendimiento bueno in lectura y escritura, habían obtenido en la pruebal un puntaje promedio significativamente más alto que los niños c'asificados con rendimiento "regular" y "deficiencia" en lectura y escritura.

En cuanto a la validez concurrente, el test hat mostrado tener una correlación de 0.62 con la prueba A.B.C. de L. Filho (1960) que como se sabe es una prueba individual que mide madurez escolar. La prucba tiene además validez de contenido.

Para esta investigación la prucba fue aplicada en 353 sujetos, de ambos sexos, entre 5 y medio y 7 y medio años. Los niños pertenccian a tres estratos seciccenómicos ${ }^{1}$ con la siguiente proporción en cada uno de ellos: Estrato Alto: $31,44 \%$; Estrato Medio: $37,40 \%$ y Estrato Bajo: $3 \mathrm{~L}, 16 \%$. Todos los sujetos cursaban el comienzo del primer año básico en el momento de la aplicación de la prucba; el test fue aplicado en abril de 1973.

Como se plantea en la introducción, la procedencia o no del jardín infantil era la variable que nos interesaban contro'ar especificamente en este estudio.

Para el'o se pensó elegir en cada estrato sociocconómico, nínos con y sin experiencia en jardín. Sin embargo, en el Estrato Alto fue piácticamente imposible encontrar niños en 1 .er año búsico sin experiencia previa en un Jardín Infantil; sólo contamos con 4 sujetos en ese sub-estrato. En los otros niveles socioeconómicos, la proporción de niños con y sin asistencia a jardín ${ }^{2}$ fue la siguiente: Estrato Medio: $82,58 \%$ con experiencia en jardín y $17,42 \%$ sin haber asistido a jardj́n; Estrato Bajo: $38,18 \%$ con y $61,82 \%$ sin asistencia a jardín respectivamente.

Aplicado el test, cuyo puntaje máximo posible es de 58 puntos, se calculó el promedio de ren-

\footnotetext{
1 De acuerdo a los Indices de Barilari y Oxley (1966).

2 Se definió "expcriemcia en jardtn" el haber asistido por lo metos un ā̄o a una instilución encaryadia de Prencscotares.
}

dimiento y desviación standard de cada subgrupo. La comparación de los promedios no sólo se hizo con cl método clásico de la prueba del "t" de Student (Guilford). Se consideró que más que una diferencia de promedios estadísticamente significativa, lo que interesaba era comprobar si los diferentes sub-grupos tenían promedios quis diferían entre sí en dos o más errores de rnedida (Adams, 1964) del instrumento utilizado, ya que en cáso contrario las diferencias pueden atribuirse a la varianza crror en la medición. En efecto, sucede muchas veces que existen diferencias significativas entre los promedios de dos o más grupos, vale decir, difcrencias que no se pucden atribuir al azar, pero la magnitud de estas diferencias en puntaje propiamente tal (ya sea puntaje observado o standard) es un valor que está comprendido en el error de medida del instrumento utilizalo. En till caso, si bien se dan diferencias significativas, ćstas sin embargo, pueden atribuirse al crror de medida propio del instrumento (error que como se sabs, siempre existe en mayor o menor grado).

Por lo anteriormente expuesto, calculamos el error de medida de la Prueba de Funciones Básicals, valor que resultó ser de $3^{3}$. Se esperaba, por lo tanto, que la diferencia entre los promedios fuera de 6 puntos o más para considerar un rendimiento diferencial entre los grupos.

Resultados. Los resultados obtenidos por los diferentes grupos, en la Prueba de Funciones Básicas, se indican en la Tabla No 1.

Antes de analizar las diferencias entre los promedios en relación al error de medida del test, tal como se planté en la metodología de este trabajo, calculamos los valores " $\mathrm{t}$ " comparando los promedios de los diferentes subgrupos entre sî.

Estos resultados se indican en la Tabla No 2.

De la tabla No 2 se puede deducir lo siguiente:

Lcs niños de nivel socioeconómico alto, que en su totalidad habían tenido experiencia de un año por lo menos en un jardín infantil, rinden igual

3 Este valor eslá en relación al puntaje bruto del test ya que las normas recien estín en claboracjon. 


\begin{tabular}{lllll}
\hline & $\begin{array}{c}\text { Nivel Medio } \\
\text { con jardin }\end{array}$ & $\begin{array}{c}\text { Nivel Medio } \\
\text { sin jardin }\end{array}$ & $\begin{array}{c}\text { Nivel Bajo } \\
\text { con jardin }\end{array}$ & $\begin{array}{c}\text { Nivel Bajo } \\
\text { sin jardin }\end{array}$ \\
\hline Nivel Alto (todos c/jardín) & $1,22(\mathrm{n} . \mathrm{s})$ & $3,06^{* *}$ & $5,00^{* *}$ & $8,25^{* *}$ \\
Nivel Med. c/jardín & - & $2,47^{*}$ & $4,26^{* *}$ & $7,22^{* *}$ \\
Nivel Med. s/jardín & - & - & 0,80 (n.s) & $\mathbf{1 , 7 5}$ (n.s) \\
Nivel Bajo con jardín & - & - & - & $\mathbf{1 , 0 2}$ (n.s) \\
\hline
\end{tabular}

$* \mathrm{p}<0,05$.

$* * \mathrm{p}<0,01$.

(n.s) no significativo.

que los niños de nivel medio con jardín $(\mathrm{t}=$ 1,22). Comparando el grupo socioeconómico alto, con los de nivel medio sin experiencia en jardín, un primer análisis parece indicar que babrían diferencias de rendimiento ya que el resultado es estadísticamente significativo $(\mathrm{t}=3,06)$ favoreciendo al grupo socioeconómico alto. Sin embargo, interpretados los resultados a la luz del error de medida del instrumento utilizado, vemos que la diferencia entre los promedios de estos dos grupos (ver tabla No 1) puede atribuirse a la varianza error del test. Por lo tanto habría que concluir en este sentido, que el grupo de niños de nivel alto no se diferencia en su rendimiento, en las tres funciones básicas analizadas del grupo socioeconómico medio, independjentemente de la experiencia o no en jardin de estos últimos.

En cambio, comparando el rendimiento de los niños del estrato sociocconómico alto, con aquéllos de nivel bajo (con o sin jardín) yemos que cl rendimiento de los primeros en la Prueba de Funciones Básicas es netamente superior ( $t=$ $5,00 \mathrm{yt}=8,25$ respectivamente).

Por otro lado, el grupo de niños de nivel socioeconómico medio, con experiencia de un año en jardin, rinde mejor que los niños de nivel bajo tanto con jardín $(\mathrm{t}=4,26)$ como sin jardín $(t=7,22)$. En cambio, los niños del estrato medio sin jardín no se diferencian en su rendimiento en el test, del grupo del estrato bajo habiendo éstos últimos asistido o no a una enseñanza prcescolar ( $\mathrm{t}=0,80 \mathrm{y} \mathrm{t}=1,75$ respectivamente).

En cuanto a las diferencias de rendimiento entre los niños de un mismo estrato, tomando la variable "experiencia en jardín" como la posible determinante de un rendimiento diferencial, sólo podemos concluir con respecto a los estratos medio y bajo ya que los niños del estrato socioeconómico alto tienen todos asistencia preescolar. Los resultados indican que ni en el estrato medio ni en el bajo, existen diferencias de puntaje entre los grupos que asistieron y los que no asistieron a jardín. En efecto, los niños del nivel medio con experiencia preescolar de un año por lo me- nos, obtienen 4 puntos más que aquéllos sin jardín. Aun cuando esta diferencia aparece estadísticamente significativa ( $t=2,47$ ) ello se puede atribuir al error de medida del instrumento. En el nivel bajo la diferencia de puntajes entre los ninos con y sin preescolaridad es menor de 2 puntos, resultado no significativo $(\mathrm{t}=1,02)$.

Este trabajo tendería a demostrar que el jardín infantil no es una experiencia suficiente para nivelar a los niños de diferentes estratos socioeconómicos, en un aspecto tan importante como sería el desarrollo de ciertas funciones básicas que han demostrado tener relación con rendimiento en lectura y escritura en primer año básico (Berdicewski-Milicic, 1974 a y 1974 b).

Se podría pensar que los jardines e'egidos en nuestri muestra tuvieran deficiencias técnicas en relación a la cstimulación de estas tres funciones. Sin embargo, como la muestra fue seleccionada de 11 colegios diferentes, es difícil pensar que todos ellos tuvieran problemas en el método de enseñanza.

Los niños de nivel modio, que tienen acceso a colegios con mayores recursos y que en muchos casos asisten al mismo colegio que los niños de nivel alto (de acuerdo a la clasificación utilizada) tampoco se diferenciaron de los niños de nivel medio que no habian asistido a jardín.

Algunos autores han sostenido que las expericncias más tempranas son las más influyentes en el desarrol!o. En nucstro grupo de niños, a pesar del jardín infantil, los stijetos del nivel bajo parten en desventaja en su aprendizaje en lectura y escritura; un año de apresto parece no ser suficientc; cn efecto, en el nivel socio-econónico bajo, el porcentaje de niños con rendimiento deficiente en lectura y escritura en primer año es mayor que en los otros niveles (Berdiccwski-Milicic, 1974 b).

De allí que parece importante el planteamiento de programas de estimulación precoz para superar la situación desventajosa de los niños.

El pediatra está en condiciones privilegiadas para intervenir en la estimulación precoz del niño 
ya que es el profesional que más tempranamente tiene contacto con él. Por otra parte, la madre habitualmente tiene una actitud de confianza abso'uta en las indicaciones médicas siendo entonces muy sensible a cualquier indicación.

Indicaciones dadas precozmente, igual que en los cuađros médicos mejoran el pronóstico del niño. En tanto que indicaciones de estimulación tardías disminuyen la efectividad terapéutica.

\section{RESUMEN}

Se plantea la importancia del Jardin Infantil en el desarrollo de tres funciones básicas: coordinación visomotora, discriminación auditiva y lenguaje. Se eligen solo estas tres funciones por contar con una prueba, construida por las autoras, y estudiada experimentalmente en Chile. La prueba es objetiva, confiable y válida para predecir rendimiento en lectura y escritura en primer año.

Se aplicó la prueba a 353 niños, de nivel socioeconómico Alto, Medio y Bajo, con y sin experiencia en jardin infantil, chyas edodes fluctúan entre 5 y medio y 7 y medio años, todos asis. tiendo al comienzo de primer año básico en el momento de aplicación del test. Los resultados indican que hay más diferencias de rendimiento entre los estratos socioeconómicos, que entre los niños de un mismo estrato. Vale decir, en un mismo estrato (Medio y Bajo) los niños que asistieron a jardin rindieron igual que los que no asistieron. Se concluye que el jardin no es una experiencia suficiente para nivelar a los niños en discriminación auditiva, lenguaje y coordinación viso-motora, funciones básicas para adquirir las desirezas de lectura y escritura. Se enfatiza la importancia de programas de estimulación precoz, anteriores al ingreso al jardin.

\section{SUMMARY}

The purpose of this paper is to emphazise the importance of pre-school experiencia in the development of the following three functions: visual-motor coordination, hearing discrimination and language. Theese three functions were selected becanse there is a test, constructeb by the authors, already applied experimentally in Chile. The test is objective, reliable and valid to predict reading and writing performance in the first year of study. The test was applied to 353 children from high, medium and low socioeconomic evels, with and without pre-school experience. The ages ranged between five and a half and seven and a half years old. All of them were attending the begining of the first grade at the moment the test was applied. Data support the hypothesis that there are more differences in performance inter socio-economic strata than inside each one. In other words, within each strata (medium and low) the children that had a pre-school experience had an equal performance than those who did not attend pre-school. It is concluded that pre-school experience is not enough to level childret in visual-motor coordination, hearing discrimination and language, which are basic functions to develop the necessary skills for reading and vriting. From here, the importance of early stimulation programs prior to the entrance of the children to pre-school experience.

\section{REFERENCIAS}

Adams, G.S. "Measurement and Evaluation in Edacation. Psychology and Guidance". Holt, Rinehart Co. Winston Inc., 1964.

Burilari-Oxley. "Evaluación del Prestigio de 90 Ocupaciones por una Muestra de Estudiantes Chilenos". Acta Psiq. y Psicol. Amer. Lat. 1966.

Berdicewski-Milicic. "Construcción de una Prueba de Funciones Básicas para predecir rendimento en primer año". Grados Académicos. Escuela de Educación, Universidad Católica de Chile, 1974 a.

Berdicewski-Milicic. "Coordinación Visomotora y Discriminación Auditiva en tres grupos de nińos de diferentes estratos socioeconómicos". Revista de Educación Diferenciada y Parvularia, Talca, Chile, 1974 b. (Por publicarse).

Bowly. "Child care and the groth of love". Penguin Books, 1965.

Fitho, L. "Los tests A.B.C.". Editorial Kapeluz, 1960.

Guilford, J.P. "Fundamental Statistics in Psychology and Education". MeGraw-Hill, 4th. Edition.

Kuder, G. "The Theory of estimation of test reliability".

Richardson, M. Psychometrica, 1937, 2, 151-160.

Lira-Rodriguez. "Diseño, realización y evaluación de un programa de estimulación precoz, para niños chilenos entre 0 y 2 años (por publicarse, 1974).

Milicic-Tschorne. "Análisis de una experiencia de docencia universitaria en los colegios de diferente estratificación social". Trabajo paria ser publicado en la Revista Latinoamericana de Psicología, 1974.

Salvat, M. "La inteligencia, mitos y realidades". Ed. Renacimiento, Barcelona, 1972.

Spitz, N. "El primer año de vida del niño. Génesis de las primeras relaciones objetos". Ed. Aguilar, Madrid, 1966. 